\title{
Switch Detection in Genetic Regulatory Networks
}

\author{
Riccardo Porreca ${ }^{1}$, Giancarlo Ferrari-Trecate ${ }^{1}$, Daniela Chieppi ${ }^{1}$, Lalo Magni ${ }^{1}$, \\ and Olivier Bernard ${ }^{2}$ \\ 1 Dipartimento di Informatica e Sistemistica, Università degli Studi di Pavia, via \\ Ferrata 1, 27100 Pavia, Italy \\ e-mail: \{riccardo.porreca, giancarlo.ferrari, daniela.chieppi, \\ lalo.magni\}@unipv .it \\ 2 INRIA, Sophia Antipolis, 2004 route des Lucioles, B.P. 93, 06902 Sophia Antipolis, \\ France \\ e-mail: olivier .bernard@inria.fr
}

\begin{abstract}
This paper considers piecewise affine models of genetic regulatory networks and focuses on the problem of detecting switches among different modes of operation in gene expression data. This task constitutes the first step of a procedure for the complete identification of the network. We propose two methods and illustrate the application to the reconstruction of switching times in data produced by a piecewise affine model of the network regulating the carbon starvation response in Escherichia coli.
\end{abstract}

\section{Introduction}

The reconstruction of biochemical networks from experimental data is nowadays recognized as one of the most important goals of systems biology. Research in this field has been promoted by the availability of experimental techniques for measuring the concentration of various molecules regulating the functioning of cells. As far as Genetic Regulatory Networks (GRN) are concerned, several measurements techniques have been developed for sampling gene expression, ranging from DNA microarrays [1] to RT-PCR and gene reporter systems [2].

We consider the problem of identifying the dynamics of GRNs using gene expression data collected with a sampling time that is sufficiently short with respect to the time constants of the network. Moreover, we restrict our attention to PieceWise Affine (PWA) models of GRNs $[3,4]$ since they are attractive under many respects. First, they preserve the essential nonlinear character of the underlying biological process. Second, they usually involve a reduced number of parameters with respect to general nonlinear models of GRNs, a feature that is appealing from the identification viewpoint. Third, powerful techniques exist for analysis and qualitative simulation of PWA models of GRNs [4].

Recently, many different algorithms have been proposed for the identification of PWA input-output models [5], and in principle they could be used for the databased reconstruction of GRNs. However, PWA systems describing GRNs possess 
a specific structure that must be preserved in order to guarantee the biological interpretability of the identified model and all existing identification methods have a limited capability of incorporating such constraints.

The data-based reconstruction of GRNs can be conceptually split in the following tasks:

1. detection of the switches in single time series of gene expression data;

2. attribution of the data to distinct modes of operation of the whole GRN (classification problem);

3. reconstruction of thresholds on concentration variables characterizing the underlying PWA model (see [4] for details) and of all combinations of thresholds consistent with the data;

4. estimation of the kinetic parameters in each mode of operation for all models generated in point 3 .

In this paper we focus on the first task in the whole identification procedure: the detection of switches in data generated by PWA input-output models of GRNs. In particular, our aim is to find switches between different modes of operation without assuming any knowledge of the model parameters. Ad hoc methods for task 2 are currently under study, while task 3 can be performed, under suitable assumptions, using the multicut algorithm proposed in [6]. Finally, task 4 can be easily carried out relying on the data classification produced in task 2 .

\section{Switch Detection in Genetic Regulatory Networks}

In the sequel we consider the problem of detecting switches in the profile of a single molecule concentration.

Consider a network involving the interaction of $n$ genes, each coding for a molecule (e.g. a protein), and denote with $\boldsymbol{x}=\left[x_{1}, \ldots, x_{n}\right]$ the vector of molecule concentrations. Due to the PWA structure of the overall model, a molecule concentration $x_{i}$ can evolve according to different modes of operation, each one characterized by an affine dynamics. Considering noisy measurements $y_{i}$ of $x_{i}$, collected with a uniform sampling time, we introduce the PWA Output Error (PWA-OE) model

$$
\left\{\begin{array}{rl}
x_{i}(k+1) & =\kappa_{i}^{j}-\gamma_{i}^{j} x \\
y_{i}(k) & =x_{i}(k)+n_{i}(k)
\end{array} \quad, \quad \text { if } \boldsymbol{x}(k) \in M_{i}^{j},\right.
$$

where $n_{i}(k) \sim W G N\left(0, \sigma_{n}^{2}\right)$. Therefore the $j$-th mode of operation is defined by the pair $\left(\kappa_{i}^{j}, \gamma_{i}^{j}\right)$ of positive rate parameters and the so-called molecule domain $M_{i}^{j}$. Molecule domains are unions of hyperrectangular regions in the state space of the overall network [7].

The first method to detect switches in a molecule concentration dynamics is based on the following switching index:

$$
o(k)=\frac{y_{i}(k+1)-y_{i}(k)}{y_{i}(k)-y_{i}(k-1)} .
$$


The index $o(k)$ enjoys the following property: in the noiseless case (i.e. $y_{i}(k)=$ $\left.x_{i}(k)\right)$ when $y_{i}(k-1), y_{i}(k)$ and $y_{i}(k+1)$ belong to the $j$-th mode, $o(k)$ takes the constant value $-\gamma_{i}^{j}$. The occurrence of a switch is therefore emphasized by time instants where $o(k)$ is not constant. A statistical analysis and suitable generalizations of $o(k)$ considering consecutive data on a time window of arbitrary length can be found in [7]. In particular, closed form expression of the $(1-\alpha)$-level confidence sets for $o(k)$ can be derived.

The algorithm based on $o(k)$ aims at aggregating data belonging to the same mode of operation. Given a set of consecutive data already aggregated, they are characterized by a switching index value and the associated confidence set $I_{M}$. Then a switching index involving the next measurement is considered, and its confidence set $I_{a}$ is computed. As a decision rule, a switch is detected if $I_{M} \cap I_{a}=\emptyset$.

A different approach is to base the aggregation of data on the estimation of the concentration dynamics within a molecule domain. In particular, the output error model (1) is estimated over the already aggregated data, and an hypothesis test is performed to assess if the next data point belongs to the same mode of operation. Under the null hypothesis $\mathrm{H}_{0}$ that $y(\bar{k}+1)$ is generated according to the model estimated over data up to $y(\bar{k})$, the confidence set $I_{a}$ for the measurement at $\bar{k}+1$ is computed. Then the aggregation of the next data point is performed if $y(\bar{k}+1) \in I_{a}$, detecting a switch otherwise.

The algorithms based on the above-mentioned switch detection techniques are described in [7]. In particular, they turn out to be complementary in the following sense: on the one hand, the method based on switching indexes outperforms the method based on nonlinear estimation for low noise levels, on the other hand, the strategy based on nonlinear filtering is the most accurate for high noise levels. These properties have been observed in [7] on the basis of extensive simulations.

\section{Switch Detection in a PWA Model of the Carbon Starvation Response of $\boldsymbol{E}$. coli}

In this section, we present the application of switch detection algorithms to data generated by the GRN regulating carbon starvation response in E. coli. For this study we considered the simplified GRN used by Drulhe et al. in [6].

The network involves interactions between genes crp, fis, gyr $A B$ and their products (proteins CRP, Fis, GyrAB), regulating the synthesis of stable RNAs. In response to a carbon starvation signal, the regulatory mechanisms inhibit the synthesis of stable RNAs and then E. coli cells abandon their exponentiallygrowth state to enter a more resistent non-growth state called stationary phase.

Data in Fig. 1 represent the reentry into exponential phase following a carbon upshift and have been generated by a PWA-OE model where the chosen noise level and sampling time are comparable with the ones found in real experiment with gene reporter systems. In Fig. 1 the real switching times and those reconstructed by applying the switching detection technique based on nonlinear 

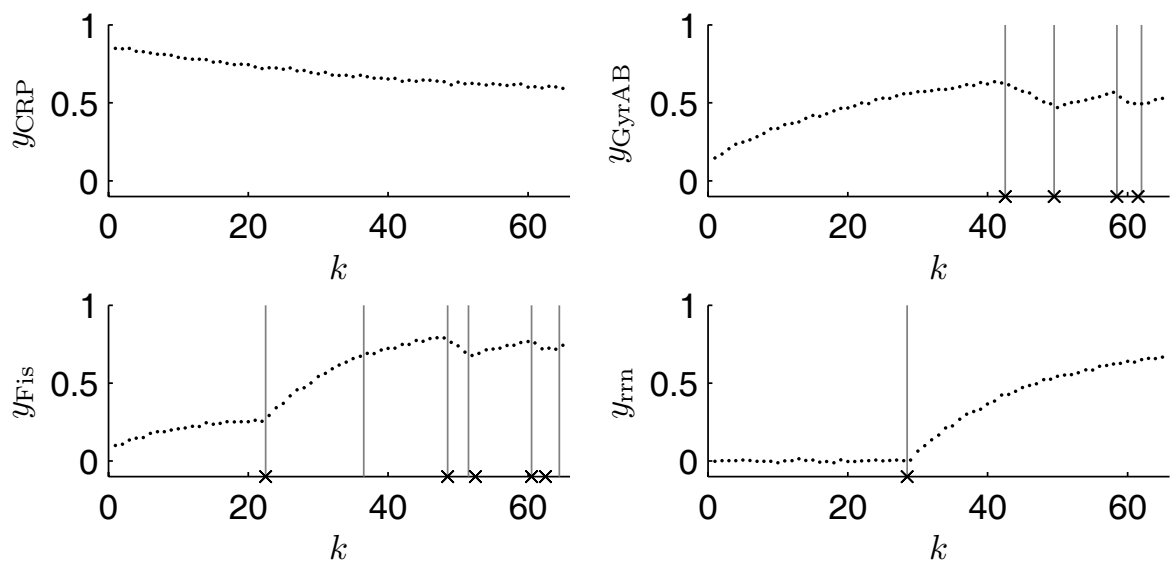

Fig. 1. Switch detection results on simulated data of the GRN regulating carbon starvation response in $E$. coli. Variables $y_{\mathrm{CRP}}, y_{\mathrm{Fis}}, y_{\mathrm{GyrAB}}$, and $y_{\mathrm{rrn}}$ denote the concentration measurements of proteins CRP, Fis, GyrAB, and stable RNAs. Vertical lines denote detected switches, while crosses correspond to real switching times.

estimation are also shown. One can observe that all switches have been identified with a satisfactory precision except for the concentration of protein Fis where a spurious switch appears.

\section{References}

1. Lockhart, D., Winzeler, E.: Genomics, gene expression and DNA arrays. Nature 405(6788) (2000) 827-836

2. Ronen, M., Rosenberg, R., Shraiman, B., Alon, U.: Assigning numbers to the arrows: Parameterizing a gene regulation network by using accurate expression kinetics. Proc. Natl. Acad. Sci. USA 99(16) (2002) 10555-10560

3. Glass, L., Kauffman, S.: The logical analysis of continuous, non-linear biochemical control networks. J. Theor. Biol. 39(1) (1973) 103-129

4. de Jong, H., Gouzé, J.L., Hernandez, C., Page, M., Sari, T., Geiselmann, J.: Qualitative simulation of genetic regulatory networks using piecewise-linear models. Bull. Math. Biol 66(2) (2004) 301-340

5. Juloski, A., Heemels, W., Ferrari-Trecate, G., Vidal, R., Paoletti, S., Niessen, J.: Comparison of four procedures for the identification of hybrid systems. In Morari, M., Thiele, L., eds.: Proc. Hybrid Systems: Computation and Control (HSCC 2005). Volume 3414 of LNCS. Springer-Verlag, Berlin (2005) 354-369

6. Drulhe, S., Ferrari-Trecate, G., de Jong, H., Viari, A.: Reconstruction of switching thresholds in piecewise-affine models of genetic regulatory networks. In Hespanha, J., Tiwari, A., eds.: Proc. Hybrid Systems: Computation and Control (HSCC 2006). Volume 3927 of LNCS. Sringer-Verlag, Berlin (2006) 184-199

7. Porreca, R., Ferrari-Trecate, G., Chieppi, D., Magni, L., Bernard, O.: Switch detection in genetic regulatory networks. Technical report, Università degli Studi di Pavia (2006) http://sisdin.unipv.it/lab/publications/TechRepSDGRN.pdf. 